\title{
Safety and Efficacy of Permacol Injection in the Treatment of Fecal Incontinence
}

\author{
Yahya A Al-Abed, Jennifer Ayers, Abraham Ayantunde, Bandipalyam V Praveen \\ Department of Colorectal Surgery, Southend University Hospital, Westcliff on Sea, United Kingdom
}

Purpose: Permacol has been gaining popularity in recent times for the treatment of fecal incontinence (FI). This study aims to evaluate the safety and efficacy of anal submucosal Permacol injection in the treatment of FI.

Methods: All consecutive patients who underwent Permacol injection for FI over a 3-year period were included. Patients' data relating to obstetric history, anorectal/pelvic operations, type of FI, preoperative anorectal physiology results and follow-up details for outcome measures were collected. Preoperative and postoperative Cleveland Clinic Florida Incontinence Scores (CCFISs) were noted. Patients were surveyed by using a telephone questionnaire to assess the quality of life and other outcome measures. Data were analysed using SPSS ver.19.0.

Results: Thirty patients ( 28 females and 2 males) with a median age of 67 years were included in the study. Of those patients, 37\%, 50\%, and 13\% were noted to have passive, mixed and urge FI, respectively. Six of the patients (20\%) had repeat Permacol injections, 5 of whom had sustained responses to the first Permacol injection for a mean of 11 months. There was a significant improvement in the CCFIS from a baseline median of 12.5, mean 12.8 (interquartile range [IQR], $6-20$ ), to a median of 3.5 , mean 4.8 (IQR, $0-20$ ), $\mathrm{P}<0.001$. Of the patients surveyed by telephone $89 \%$ were satisfied with their overall experience and the improvement in their symptoms following Permacol injections.

Conclusion: This study has demonstrated that Permacol injection for the treatment of FI is safe and effective and has no associated major complications. However, the results are not permanent; consequently, a significant proportion of the patients with an initial response may require repeat injections.

\section{Keywords: Fecal incontinence; Permacol injection; Anal bulking agent}

\section{INTRODUCTION}

Fecal incontinence (FI) is the involuntary loss of rectal contents through the anal canal $[1,2]$. It is believed to be underreported by patients and underrecognised by clinicians. FI affects up to $8 \%$ of the adult population over the age of 65 years and up to $50 \%$ of nursing home residents $[3,4]$. FI often has several contributory factors; therefore, diagnosing the cause or causes for each individ-

Received: January 18, 2016 - Accepted: March 2, 2016

Correspondence to: Yahya A Al-Abed, M.D.

Department of Colorectal Surgery, Southend University Hospital, Prittlewell Chase, Westcliff-on-Sea, SSO ORY, United Kingdom

Tel: +44-1702-435555, Fax: +44-1702-385833

E-mail:yalabed@yahoo.co.uk

(C) 2016 The Korean Society of Coloproctology

This is an open-access article distributed under the terms of the Creative Commons Attribution NonCommercial License (http://creativecommons.org/licenses/by-nc/4.0) which permits unrestricted noncommercial use, distribution, and reproduction in any medium, provided the original work is properly cited. ual in important. Appropriate treatment of this condition relies on accurate diagnosis and careful patient selection. The aims of the treatment strategies are to improve anal continence and quality of life and, when complete treatment is not achievable, to help patients to cope with their symptoms. The recent National Institute for Clinical Excellence (NICE) guidance in the United Kingdom on FI [5] emphasises the need for patients to have access to healthcare professionals with relevant training, skills, and experience. However, many healthcare professionals do not have expertise in the diagnosis and the treatment of FI and may only provide advice on techniques and products that can be used to manage the the condition.

FI can be classified as urge or passive according to the symptoms presented by the patient. Since the introduction of endoanal ultrasonography, our understanding of sphincter function and the mechanisms of incontinence have greatly increased. It is now understood that defects in the internal anal sphincter (IAS) result in passive FI. In contrast, defects in the external anal sphincter (EAS) 
result in impairment in voluntary anal contraction and, hence, in urge FI $[6,7]$.

The treatment of FI is challenging and there are various treatment options depending on the aetiological or contributing factors [8]. The first line of treatment for passive FI is the use of stool bulking agents, antidiarrhoeal agents and biofeedback. Surgical alternatives may be considered following the failure of these conservative measures, but they are invasive and the results are generally not encouraging [9]. However, degenerate or disrupted IAS is believed not to be easily amenable to surgical repair and is associated with poor results [10].

There is now published evidence from studies showing improvement in the objective and the subjective symptoms of perianal soiling and passive incontinence by the use of submucosal injection of bovine collagen or Permacol (Tissue Science Laboratories, Aldershot, Hampshire, UK) in the areas of the anal cushions $[9,11-16]$. However, a recent Cochrane review only found four eligible randomised controlled trials of variable quality and concluded that there was no robust evidence due to the limited number of identified trials and their methodological weaknesses, but a trend towards improvement in the short term was noted [17]. Thus, the aim of this study was to evaluate our experience with the safety and efficacy of Permocol injection as an anal bulking agent in the treatment of FI in a cohort of patients.

\section{METHODS}

This study, which was registered and approved by our hospital's clinical audit department (research and development) as an audit of practice, included consecutive patients who had undergone Permacol injections, an anal bulking agent for the treatment of FI, at our institution over a 3-year period (March 2010 to March 2013). All patients with a history of FI were evaluated according to our standard management protocol in a dedicated pelvic floor disorders clinic with physical examinations including digital rectal examination and rigid sigmoidoscopy followed by anorectal physiology tests, pudendal nerve latency time measurements and endoanal ultrasonography. Flexible sigmoidoscopy and/or colonoscopy were selectively performed where indicated. Patients were selected for treatment with Permacol injection anal bulking agent according to the local treatment algorithm in line with NICE guidelines. These patients had failed conservative therapy such as diet, fibre/bulking agent therapy, antidiarrhoeal medications and had presented with evidence of abnormalities of the IAS on an endoanal ultrasound scan (EUS). The procedure was performed by a single colorectal surgeon who had a special interest in FI and had been trained in this technique.

Data collected included patients demographics, clinical data relating to obstetric history, anorectal/pelvic operations, type of FI, preoperative anorectal physiology study results, operative findings and follow-up details for outcome measures. All patients were followed-up two months after Permacol injection and every 3 months thereafter. Preoperative and postoperative Cleveland Clinic Florida Incontinence Scores (CCFISs) were calculated for each patient. The CCFIS was determined in all patients at their first clinical assessment in the outpatient clinic and at their first clinic follow-up appointment after Permacol injection. A CCFIS of zero indicates complete continence while a score of 20 indicates severe incontinence. The difference between the pre- and the postinjection scores was calculated, and the treatment was considered to have been successful for patients showing a $50 \%$ or more reduction in their scores.

\section{Follow-up protocol}

The first follow-up review was usually about 8 weeks post procedure, with additional follow-ups at 3, 6, 9, and 12 months, in the dedicated pelvic floor disorders clinic, at which times the patient's response to the injections was recorded and the CCFIS was calculated. All patients were surveyed through a telephoning questionnaire to assess the quality of life and other patient outcome measures. Clinical responses to Permacol injections were classified as complete, partial and no response. Complete response was defined as complete resolution of the symptoms with no or one or less episode of FI per month. Partial response was defined as a significant improvement of symptoms with reductions of more than $50 \%$ in the number of episodes of FI per month.

All collected data on patients were analysed using IBM SPSS Statistics ver. 19.0 (IBM Co., Armonk, NY, USA). Continuous variables were expressed as means plus or minus standard deviations (SDs) or ranges and medians. The paired t-test was used to test the significance of the difference in CCFIS pre-and postinjections. A P-value of $\leq 0.05$ was deemed significant.

\section{Procedure}

The procedure was performed under general or regional anaesthesia (GA or RA) in the lithotomy position usually as a day case. A preoperative enema is desirable in order to provide a relatively clean anorectal site of injection and to prevent a subsequent hard stool from compressing the implant postinjection. A single dose of appropriate prophylactic antibiotic (Augmentin/Metronidazole) was given at the induction of anaesthesia.

The Permacol injectable bulking agent was mixed by attaching an empty syringe to a prefilled syringe containing the agent and by passing the solution back and forth between the two syringes 20 times to achieve adequate mixing. The mixed Permacol was then attached to a green $(21 \mathrm{G})$ needle. The very gentle use of an Eisenhammer retractor afforded an excellent view of the anal canal and the distal rectum to locate the desirable site of injection. With the patient in the preferred position and the Eisenhammer retractor exposing the anorectal canal, the internal sphincter was identified, and three submucosal injections were administered, one each at the 3, 7, and 11 oclock positions corresponding to the positions of the anal cushions. Permacol was injected starting just below the dentate line and mainly above it to produce a bulge in 
the submucosal layer. We normally injected the Permacol in a fanning pattern at these three positions in such a way that the ultimate bulges produced a funnel anal canal with an increasing anorectal angle. We usually injected about $2.5 \mathrm{~mL}$ of Permacol at each site. A more tailored injection was done in some patients to inject Permacol at the site of the IAS defect or deficiency as shown on the preoperative EUS. Steps were taken to ensure that the needle remained in the injection tract for about $10-15$ seconds at the completion of injection. After the needle had been withdrawn, the injection point was covered with a piece of gauze upon which finger pressure was applied for 30 seconds. These measures were to prevent the leakage of the bulking agent. Adequate care was exercised to prevent spillage of Permacol through the mucosa into the anorectal canal.

Patients were allowed to go home the same day once they had fully recovered from anaesthesia in accordance with outpatient

Table 1. Patients' demographic and clinical data

\begin{tabular}{|c|c|}
\hline Variable & Value \\
\hline Age (yr), median (range) & $67(52-87)$ \\
\hline \multicolumn{2}{|l|}{ Sex } \\
\hline Male : female & $2: 28$ \\
\hline MMRP, median (range) & $37(10-116)$ \\
\hline MMSP, median (range) & $50(5-90)$ \\
\hline \multicolumn{2}{|l|}{ Obstetric history $(\mathrm{n}=28)$} \\
\hline \multicolumn{2}{|l|}{ Vaginal delivery } \\
\hline Yes : no & $21: 7$ \\
\hline \multicolumn{2}{|l|}{ Forceps delivery } \\
\hline Yes : no & $4: 24$ \\
\hline \multicolumn{2}{|l|}{ Episiotomy } \\
\hline Yes : no & $9: 19$ \\
\hline \multicolumn{2}{|l|}{ Perineal tear } \\
\hline Yes : no & $9: 19$ \\
\hline \multicolumn{2}{|l|}{ Type of incontinence } \\
\hline Urge & 4 \\
\hline Passive & 11 \\
\hline Mixed & 15 \\
\hline Delayed PNMTL, n (\%) & $16 / 30(53)$ \\
\hline \multicolumn{2}{|c|}{ Abnormal sphincter morphology } \\
\hline IAS thinned/degenerated & 18 \\
\hline IAS disrupted & 6 \\
\hline EAS thinned/degenerated & 10 \\
\hline EAS disrupted & 9 \\
\hline Repeat injections, n (\%) & 6/30 (20) \\
\hline
\end{tabular}

MMRP, maximum mean resting pressure; MMSP, maximum mean squeeze pressure; PNMTL, pudendal nerve motor latency; IAS, internal anal sphincter; EAS, external anal sphincter. surgery protocol. Patients were prescribed mild laxatives to prevent constipation and avoid straining. No immediate postprocedure complications or adverse events were recorded.

\section{RESULTS}

A total of 30 patients ( 28 females and 2 males) with a median age of 67 years (range, 52-87 years) were treated for FI with Permacol injection anal bulking agent during the study period. Patients' demographics, as well as their clinical and obstetric data, are shown in Table 1. Passive FI was noted in 11 patients (37\%), 15 patients had mixed FI (50\%) while only 4 patients (13\%) had urge FI. Six out of the 30 patients (20\%) had repeat Permacol injections, 5 of whom had an initial complete response to the injection and 1 of whom had a partial response. These patients had sustained response to the first Permacol injection for a mean of 11 months (interquartile range [IQR], 5-24 months). The baseline pretreatment anorectal physiology test parameters showed a median maximum mean resting pressure of $37 \mathrm{~cm}$ of water (IQR, 10-116) and a median maximum mean squeeze pressure of $50 \mathrm{~cm}$ of water (IQR, 5-90).

An objective assessment of the improvement after Permacol injection was carried out using the CCFIS. A significant improvement in CCFIS from a baseline median of 12.5 , mean 12.8 (IQR, 6-20), to a median of 3.5, mean 4.8 (IQR, $0-20$ ), P $<0.001$, at a median of 8 weeks post initial injection was noted (Figs. 1-3). The mean percentage change in the CCFIS score was $63.5 \%$, with a median of $66 \%$ (IQR, $0 \%-100 \%$ ). The percentage change in the CCFIS was greater for patients with passive and mixed types of FI and less, but still significant, in patients with urge FI $(\mathrm{P}=0.091)$ (Fig. 4).

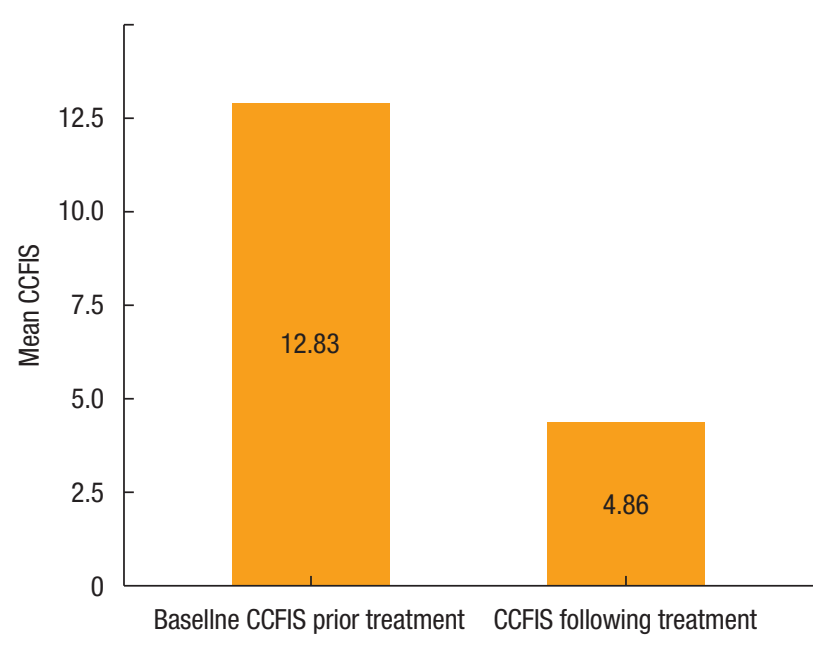

Fig. 1. Mean Cleveland Clinic Florida Incontinence Score (CCFIS) prior to and at 12 weeks post Permacol (Covidien, UK) injections. $\mathrm{P}<0.001$. 


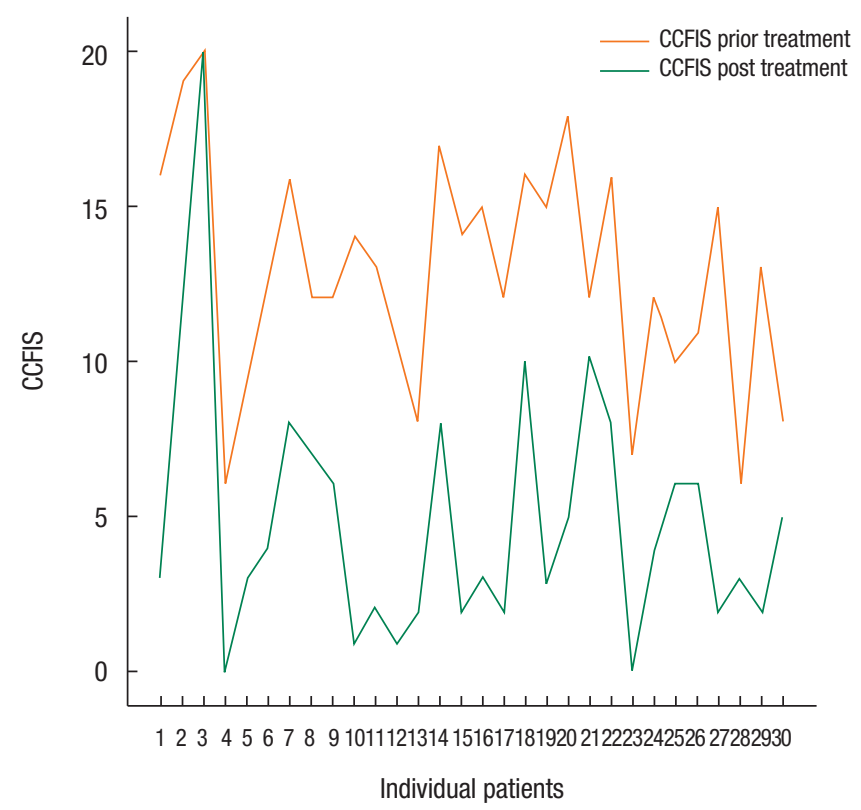

Fig. 2. Cleveland Clinic Florida Incontinence Score (CCFIS) prior to and at 6-12 weeks post Permacol (Covidien, UK) injections.

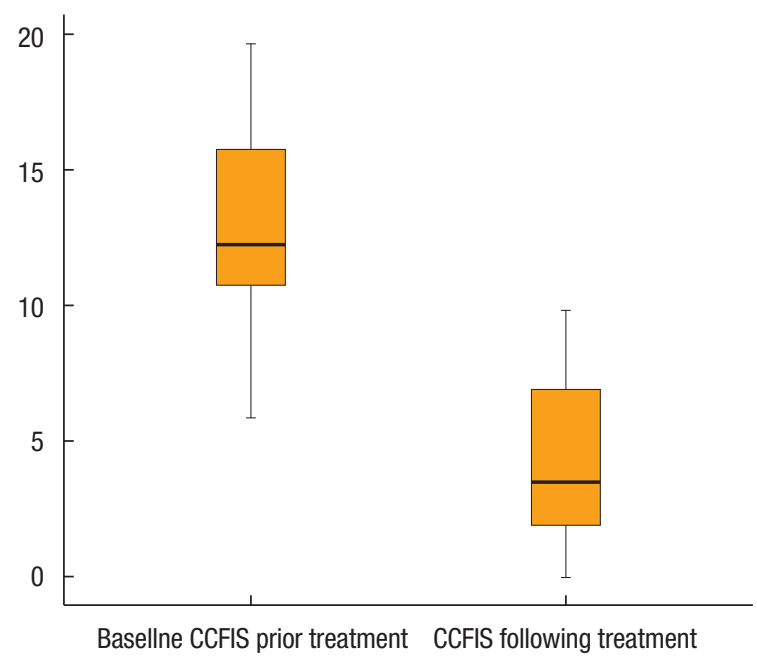

Fig. 3. Cleveland Clinic Florida Incontinence Scores (CCFISs) and means prior to and following Permacol (Covidien, UK) injections (paired t-test).

\section{Outcomes obtained by using a telephone questionnaire survey}

Eighteen of the 30 patients responded to the telephone questionnaire survey, giving a response rate of $60 \%$. Twelve patients did not respond due to 1 having passed away, 1 having had a tracheostomy, and 10 having relocated with no traceable telephone contact. The mean time between Permacol injection and the telephone questionnaire survey was 20.4 months (IQR, 3-36 months). Patients' satisfaction with the anal bulking agent in con-

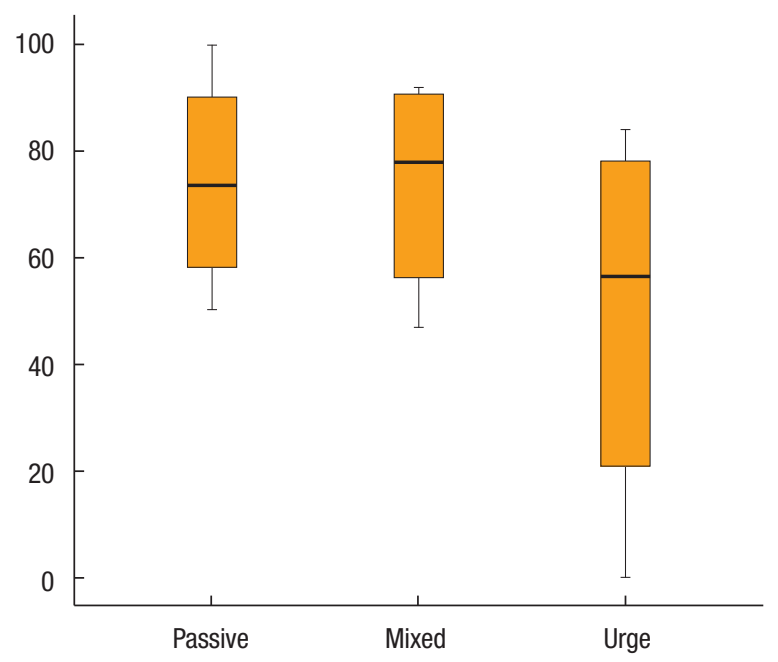

Fig. 4. Percentage change in Cleveland Clinic Florida Incontinence Scores following Permacol (Covidien, UK) injection according to the different types of fecal incontinence.

Table 2. Overall patients' satisfaction with Permacol ${ }^{\mathrm{a}}$ injection

\begin{tabular}{lll}
\hline Score out of 10 & \multicolumn{1}{c}{ Satisfaction rate } & No. of patients (\%) \\
\hline $8-10$ & Very satisfied & $9(50)$ \\
$6-7$ & Satisfied & $7(39)$ \\
$4-5$ & Neither satisfied nor dissatisfied & $0(0)$ \\
$2-3$ & Dissatisfied & $0(0)$ \\
$0-1$ & Very dissatisfied & $2(11)$ \\
\hline
\end{tabular}

Eighty-nine percent of the patients were either satisfied or very satisfied with the outcome.

aPermacol (Covidien, UK).

trolling their symptoms was scored by using a visual analogue scale from 1 to 10 . The median satisfaction score was 7.5. Half of the 18 patients were very satisfied with their overall experience and with the improvement in their symptoms following Permacol injections. Thirty-nine percent (7/18) of the patients were satisfied with the outcome, and only $11 \%$ (2 of 18 ) were dissatisfied. Therefore, $89 \%$ of our patients appear to have been either very satisfied or at least satisfied with the outcome following Permacol injections (Table 2).

Thirty-three percent (6 of 18) of the surveyed patients experienced some pain at the injection site requiring mild analgesics while 17\% (3 of 18) had minimal rectal bleeding. None of these complications required a visit to the hospital. No associated anorectal or perineal infection was reported following Permacol injection. Of the patients responding to the telephone questionnaire, $89 \%$ (16 of 18) would recommend Permacol injection to a friend with similar FI symptoms, and 78\% (14 of 18) would be happy to undergo a repeat Permacol injection if advised to do so by their surgeon. 
Table 3. Patients' response to treatment at first follow-up following Permacol $^{\mathrm{a}}$ injection $(\mathrm{n}=30)$

\begin{tabular}{lc}
\hline Response & No. (\%) \\
\hline No response (no improvement in symptoms) & $2(6)$ \\
Partial response (reduction in the number of Fl episodes per month) & $11(37)$ \\
Complete response (No or $\leq 1$ episode of Fl per month) & $17(57)$ \\
\hline
\end{tabular}

aPermacol (Covidien, UK).

Patients' subjective responses to treatment, as evident by improvements in the FI symptoms, were recorded at the 8-week follow-up, and up to $57 \%$ of patients in our cohort had complete responses to treatment. Partial responses were noted in $37 \%$ of patients, and only $6 \%$ had no response or minimal change in their pre-injection symptoms (Table 3).

\section{DISCUSSION}

FI not only causes significant patient morbidity and psychosocial disturbances, but also poses a large challenge to the professionals involved in its treatment. Over the last 2 decades, since the first reports in 1993 on using anal bulking agents for the treatment of FI [18], several reports and studies have been published using different types and techniques of anal bulking with variable results and mixed outcomes. Recently, more studies have been published; the most recent of which had investigated the long-term effectiveness of Permacol injection for the treatment of passive FI [9]. In that study with a follow-up duration of a minimum of 36 months, the authors showed that Permacol injection for the treatment of FI was simple, safe and effective in the short, mid, and long terms. However, repeat injections were required in $50 \%$ of patients after a median of 18 months. These results were similar to the ones noted in earlier smaller studies on Permacol injection.

The results of our current study are in keeping with those in the published literature. We have demonstrated that Permacol anal bulking injections provide an efficient and safe solution for selected patients with FI. This was shown from the improvement in the CCFIS and from the responses to telephonic interviews designed to assess the patients' quality of life. Almost $90 \%$ of our patients were satisfied or highly satisfied with the results of Permacol injections with regards to its long-lasting results and the absence of major complications.

With regards to safety of anal bulking implants, most studies report no major adverse outcomes. The commonly-reported adverse effects were pain at the injection site and leakage of the implant material. In our series, we did not encounter any significant complications following the injections. This was evident from the patients' outcomes reported during telephone interviews. Only $33 \%$ of our patients that were interviewed reported pain at the injection site, which was controlled with simple analgesics, and $17 \%$ reported minimal bleeding, which did not require visiting the hospital.
According to the Leeds group [9], the CCFIS significantly improved following Permacol injection. They demonstrated that this effect was sustained for at least six months before it started to gradually deteriorate by 12 months. Similarly, Hussain et al. [13] demonstrated improvement in St Mark's incontinence scores at 1 year, with a decline in this improvement at 2 years. This effect was also noted by the St Mark's group with a small number of patients [14]. In our case series, we demonstrated a significant improvement in the CCFISs at a median of 8 weeks (range, 6-12 weeks) following injection. The scores were significantly reduced from a mean of 12.8 to a mean of $4.8(\mathrm{P}=0.0001)$. This effect was sustained for a mean of 11 months (range, 5-24 months). These results are in keeping with the previously published results on Permacol anal bulking injections.

That injectable anal bulking agents can be used for treating passive FI occurring due to IAS pathology is widely accepted. In our retrospective study, Permacol injections were offered mainly to patients with passive and mixed FI (37\% and 50\%, respectively) and only to a few patients with urge incontinence (13\%). The majority of the patients appear to have had improvements in their symptoms as shown by the improvements in their CCFISs. The changes in the CCFISs were greater in the passive and mixed groups and were less, but considerable, in the urge group. Three out of 4 patients with urge incontinence still experienced significant improvement. We feel that this result is important, despite the small number of patients. In our study, all patients with urge FI had underlying abnormalities involving both IAS and EAS, which might explain the improvements in their symptoms following injections. Currently, a multicenter, blinded, randomized controlled trial is taking place in Norway and involves patients for treatment of FI resulting from obstetric anal sphincter injuries (the KISS trial) [19]. Patients are being randomised for treatment with either sacral nerve modulation or Permacol anal bulking injections. All patients, regardless of the type of incontinence, are included according to the inclusion criteria. Results of this trial could prove the benefits of Permacol injections in patients with all types of FI.

In our study, we were able to demonstrate subjective improvements in the patients' symptoms by using our telephone questionnaire. We minimised the risk of study bias by allowing the telephone interview to be conducted by a clinician who was not involved in the treatment at any stage. In this telephone interview, we assessed the experience of the patients regarding the Permacol injection and their perception of the treatment response. This interview showed that $89 \%$ of patients were at least satisfied with the outcomes following Permacol injections, which was complemented by the high positive response rates when they were asked if they would repeat the injections if advised to do so and if they would recommend this treatment to a family member or close friend. To our knowledge, we have the largest series of FI patients treated with Permacol injections whose subjective outcomes were measured by telephonic interviews. 
Like other retrospective studies, our study has strengths and limitations. Although this study is not a randomised controlled trial, we believe that we have demonstrated results in agreement with the current evidence available in the literature. We believe that this study adds more weight to the available evidence on anal bulking injections for the treatment of FI. Our study design included objective, as well as subjective, variables to support the evidence for the efficacy and the safety of Permacol anal bulking injections. Our cohort of patients also involved a few cases of urge FI, and the results for this group provided some new insight.

Since the publication of NICE guidance on the safety and the efficacy of anal bulking injections and the American Society of Colon and Rectal Surgeons (ASCRS) parameters for the treatment of FI [20], an increasing number of reports have been published on anal bulking agents and on Permacol injection, in particular. These reports, along with our own experience, have demonstrated that Permacol injection is safe and efficient for the treatment of FI. We, therefore, suggest re-evaluation of the NICE and the ASCRS guidance to take into account the recent evidence and to allow wider and safer use of this effective treatment.

We know from previous reports that repeat injections are needed in a significant number of patients, and in our experience, this was required in at least one-fifth of our cohort after a mean of 11 months, in keeping with the observations in reports in the literature. This is important to help patients make informed decisions on their treatment. This is particularly important for clinicians during discussions of the treatment options and counselling and when obtaining informed consent.

In conclusion, our study has demonstrated that Permacol injection for the treatment of FI is a safe procedure and is not associated with any major complications. The overall success rate in reducing FI episodes is encouraging. The results are not permanent, so a significant percentage of patients responding to this will require repeat injections. This is important while counselling and obtaining consent from the patients. Permacol injection for FI is associated with a high patient satisfaction rate in the short and medium terms. A prospective randomized clinical trial is required to further evaluate the role of Permacol injection in the treatment of FI.

\section{CONFLICT OF INTEREST}

No potential conflict of interest relevant to this article was reported.

\section{REFERENCES}

1. Bharucha AE, Blandon RE. Anatomy and physiology of continence. In: Ratto C, Doglietto GB, editors. Fecal incontinence diagnosis and treatment. Milan (IT): Springer; 2007. p. 3-12.

2. Matzel KE. Surgery for fecal incontinence. In: Zbar AP, Wexner SD, editors. Coloproctology. London: Springer; 2010. p. 109-20.

3. Perry S, Shaw C, McGrother C, Matthews RJ, Assassa RP, Dallos- so $\mathrm{H}$, et al. Prevalence of fecal incontinence in adults aged 40 years or more living in the community. Gut 2002;50:480-4.

4. Nelson R, Norton N, Cautley E, Furner S. Community-based prevalence of anal incontinence. JAMA 1995;274:559-61.

5. National Institute for Health and Clinical Excellence. Fecal incontinence in adults: management [Internet]. London: NICE, 2007 [cited 2013 Jun 3]. Available from: https://www.nice.org.uk/guidance/cg49.

6. Vaizey CJ, Kamm MA, Bartram CI. Primary degeneration of the internal anal sphincter as a cause of passive fecal incontinence. Lancet 1997;349:612-5.

7. Santoro GA, Di Falco G. Benign anorectal diseases. diagnosis with endoanal and endorectal ultrasound and new treatment options. Berlin: Springer; 2006.

8. Tan JJ, Chan M, Tjandra JJ. Evolving therapy for fecal incontinence. Dis Colon Rectum 2007;50:1950-67.

9. Maslekar S, Smith K, Harji D, Griffiths B, Sagar PM. Injectable collagen for the treatment of fecal incontinence: long-term results. Dis Colon Rectum 2013;56:354-9.

10. Morgan R, Patel B, Beynon J, Carr ND. Surgical management of anorectal incontinence due to internal anal sphincter deficiency. Br J Surg 1997;84:226-30.

11. Vaizey CJ, Kamm MA. Injectable bulking agents for treating fecal incontinence. Br J Surg 2005;92:521-7.

12. Chattopadhyay D. Green S. Anal bulking with Permacol injection injection to treat passive fecal incontinence: medium term results. Colorectal Dis 2009;11(Suppl 1):15-45.

13. Hussain ZI, Lim M, Mussa H, Abbas K, Stojkovic S. The use of Permacol injections for the treatment of fecal incontinence. Updates Surg 2012;64:289-95.

14. Maeda Y, Vaizey CJ, Kamm MA. Pilot study of two new injectable bulking agents for the treatment of fecal incontinence. Colorectal Dis 2008;10:268-72.

15. Smart N, Aspey H, Richardson D, Plusa S, Mercer-Jones M. Submucosal injections with Permacol injection injection for fecal incontinence. Colorectal Dis 2005;7(Suppl S2):59.

16. Hussain ZI, Lim M, Stojkovic SG. Systematic review of perianal implants in the treatment of fecal incontinence. Br J Surg 2011; 98:1526-36.

17. Maeda Y, Laurberg S, Norton C. Perianal injectable bulking agents as treatment for fecal incontinence in adults. Cochrane Database Syst Rev 2010;(5):CD007959.

18. Shafik A. Polytetrafluoroethylene injection for the treatment of partial fecal incontinence. Int Surg 1993;78:159-61.

19. University Hospital of North Norway. Treatment of fecal Incontinence after obstetric anal sphincter injuries (KISS) [Internet]. Bethesda (MD): U.S. National Institutes of Health; 2012 [cited 2013 Apr 10]. Available from: http://clinicaltrials.gov/show/ NCT01528995.

20. Tjandra JJ, Dykes SL, Kumar RR, Ellis CN, Gregorcyk SG, Hyman NH, et al. Practice parameters for the treatment of fecal incontinence. Dis Colon Rectum 2007;50:1497-507. 